\title{
Repositioning of drugs using open-access data portal DTome: A test case with probenecid (Review)
}

\author{
MOHAMMAD U. AHMED, DYLAN J. BENNETT, TZE-CHEN HSIEH, \\ BARBARA B. DOONAN, SABA AHMED and JOSEPH M. WU
}

Department of Biochemistry and Molecular Biology, New York Medical College, Valhalla, NY 10595, USA

Received August 11, 2015; Accepted October 12, 2015

DOI: $10.3892 / \mathrm{ijmm} .2015 .2411$

\begin{abstract}
The one gene-one enzyme hypothesis, first introduced by Beadle and Tatum in the 1940s and based on their genetic analysis and observation of phenotype changes in Neurospora crassa challenged by various experimental conditions, has witnessed significant advances in recent decades. Much of our understanding of the association between genes and their phenotype expression has benefited from the completion of the human genome project, and has shown continual transformation guided by the effort directed at the annotation and characterization of human genes. Similarly, the idea of one drug-one primary disease indication that traditionally has been the benchmark for the labeling and usage of drugs has also undergone evident progressive refinements; in recent years the science and practice of pharmaceutical development has notable success in the strategy of drug repurposing. Drug repurposing is an innovative approach where, instead of de novo synthesis and discovery of new drugs with novel indications, drug candidates with the desired usage are identified by a process of re-profiling using an open-source database or knowledge of known or failed drugs already in existence. In the present study, the repurposing drug strategy employing open-access data portal drug-target interactome (DTome) is applied to the uncovering of new clinical usage for probenecid.
\end{abstract}

\section{Contents}

1. Introduction

2. Gout

3. Probenecid as a uricosuric drug

4. Repositioning probenecid using DTome analysis

5. Repositioning of probenecid as an antiviral drug

6. Repositioning of probenecid as an anticancer agent

7. Conclusion

Correspondence to: Dr Joseph M. Wu, Department of Biochemistry and Molecular Biology, New York Medical College, 15 Dana Road, Basic Sciences Building, Room 147, Valhalla, NY 10595, USA

E-mail: joseph_wu@nymc.edu

Key words: drug repurposing, drug-target interactome analysis, probenecid

\section{Introduction}

Drug repositioning has become an increasingly attractive alternative to traditional drug development. Two major factors have contributed to this change, specifically cost and time. Traditional drug development consists of three stages: Discovery, preclinical and clinical testing. Each phase faces unique challenges. The drug is first tested for efficacy using in vitro or in silico modeling against specific targets in the discovery stage. The drug is subsequently tested with animal models in the preclinical stage, and human testing is conducted in the first clinical stage. While certain drugs in development may appear promising, there is no guarantee the drug will be effective in humans (1). Approximately $60 \%$ of the new oncology drugs under development throughout the 1990's have successfully completed phase 2 programs and subsequently failed in phase 3 clinical trials. Only $5 \%$ of all agents developed for oncology applications in this time period have ever reached application (2).

The cost of drug development has ballooned within the United States (US) over the past decade, rising from $\$ 26$ billion in 1998 to $\$ 50$ billion in 2008 (3). The increase in cost is due in part to the increase in time required to develop a drug and secure approval from the US Food and Drug Administration (FDA); since the 1990's this time period has increased to 15 years (1). Further contributing to high development costs is the increasingly strict regulatory environment imposed by the FDA, resulting in high attrition rates for drugs in development. Between 1999 and 2004, the probability of a drug in the clinical stage reaching the market was only $16 \%$. The drug industry in general has become an increasingly unmanageable environment (3).

In contrast to traditional drug development, the process of repositioning, repurposing, redirecting or reprofiling of existing approved drugs involves discovering uses beyond their initial medical applications (4). Such an approach has previously been commonly known as 'off-label' use, such as when physicians make a decision to prescribe a particular drug for unapproved indications, age groups, dosages or forms of administration. Repositioning of existing and marketed drugs reduces safety and pharmacokinetic uncertainty due to the extensive pharmacokinetic and toxicological data already available (5). Reliable information also exists in formulation, bioactivities and pharmaceutical properties, such as absorption, 
distribution, metabolism, excretion and toxicity. As a result, the development process can be reduced to just 3-12 years (4). Knowledge of the traditional dosing and therapeutic profile of the repositioned drugs can be readily integrated with current scientific advances to provide a more informed framework on how patients may benefit from these drugs and anticipation of untoward side effects.

Another benefit of drug repositioning research and development is that it can accurately predict new drug targets as primary sites of action, and also identify off-targets and mediators of side effects (6). Campillos et al (6) compared side-effect information between unrelated drugs to infer common protein targets. It was experimentally concluded that drugs sharing a similar chemical structure and similar side effects likely bind to associated targets, sometimes beyond the drug's therapeutic indication. By contrast, Keiser et al (7) predicted new molecular targets of drugs by comparing the similarity of the ligands targeted. The advantage of their method is its ability to capture similarities between drugs that are otherwise dissimilar in chemical structure. For example, Rescriptor was indicated to bind the histamine $\mathrm{H} 4$ receptor, a target unrelated to its therapeutic function as a reverse transcriptase inhibitor, but was consistent with the painful rashes associated with the use of Rescriptor.

Drug repositioning studies can also compare gene expression between drugs and disease states. Lamb et al (8) illustrated the value of a large publicly available database known as the 'Connectivity Map' that could be used to study the associations between drugs, genes and disease. The Connectivity Map can be applied without the use of extensively precise experiments. Using this method, Sirota et al (9) hypothesized that a drug has a potential therapeutic use for a disease state and a drug created opposite patterns of gene expression. Cimetidine's primary therapeutic use was to treat stomach acidity and peptic ulcers due to its antagonism of the histamine $\mathrm{H} 2$ receptor; however, the Connectivity Map showed that cimetidine could be used to treat lung adenocarcinoma. This was confirmed experimentally through a statistically significant reduction in tumor size. Thus, exploring gene expression to characterize disease and drug signature is a viable method to reposition drugs. Given the wealth of evidence showing that convergent signaling pathways can elicit divergent downstream cellular events in different diseased states, the potential for repurposing existing drugs for novel therapeutic indications appears immeasurable.

There are numerous examples of the success of drug repositioning, but a notable example is the repurposing of sildenafil. Sildenafil was developed by Pfizer to treat angina via inhibition of phosphodiesterase-5 (4). Although sildenafil proved to be ineffective in treating angina, researchers observed that inhibition of phosphodiesterase- 5 resulted in erections among male subjects (4). Following this discovery, Pfizer repositioned and successfully remarketed sildenafil under the brand name Viagra, as a treatment for erectile dysfunction (4).

The present review focuses on the use of the drug-target interactome (DTome) database to illustrate the developing field of drug repositioning, as applied to probenecid, previously known as Benemid. Probenecid was initially introduced to slow the elimination of the antibiotic penicillin via renal tubular secretion $(10,11)$. In the 1950's and 1960's the 'Probenecid Test' was used as a clinical tool to diagnose depression and psychiatric disorders $(10,11)$. Currently, probenecid is primarily used as a long-term therapy for patients with refractory gout or in patients who are unresponsive to first-line treatments (12). It is also used in the laboratory to investigate intracellular calcium within in vitro cells (11). However, new evidence suggests that probenecid may have other therapeutic potentials. The traditional therapeutic target of probenecid in the treatment of gout will first be reviewed, followed by an overview on the potential for repositioning probenecid as an anticancer and anti-viral drug by exploring drug-protein and secondary protein-protein interactions.

\section{Gout}

Gout is a metabolic disorder characterized by chronic elevation in uric acid levels $>6.8 \mathrm{mg} / \mathrm{dl}$. A sustained increase in uric acid causes hyperuricemia and results in the formation and deposition, preferentially in joints, of monosodium urate monohydrate crystals, otherwise known as tophi (13). Upon shedding, tophi are believed to cause intense pain by triggering acute but self-limiting joint inflammation (14). It is estimated that the prevalence of gout has risen to $3.9 \%$ from $2.7 \%$ between 1988 and 1994 based on the National Health and Nutrition Examination Survey (15).

Gout has been proposed to progress in four stages (Fig. 1) (16). Stage 1 is characterized by asymptomatic hyperuricemia that has the potential to develop into gout. Acute gout flairs are classified as stage 2 . In stage 3 , periods are susceptible to additional flairs and otherwise asymptomatic hyperuricemia prevail, whereas establishment of stage 4 is marked by the presence of chronic gout. The objective of short-term therapy of gout is to manage acute episodes and minimize the joint inflammation observed in stage 2. A popular short-term treatment is colchicine, a non-selective inhibitor of inflammasome activity and interleukin-1 production in leukocytes and migration of leukocytes to affected areas, as well as release of histamine from mast cells (14). In contrast to short-term treatments, long-term therapy manages patients in stages 3 and 4 .

Hyperuricemia and gout can be attributed to the overproduction, underexcretion or increased intake of urate (16). As shown in Table I, urate-lowering therapy (ULT) includes administration of drugs that act to prevent uric acid formation, e.g., allopurinol or febuxostat, or enhance its excretion, such as probenecid, as well as changes in the diet and lifestyle. ULT drugs are prescribed as necessary to achieve non-hyperuricemic blood plasma levels (17).

Allopurinol, a purine analogue that lowers the production of uric acid by competitively inhibiting the enzyme xanthine oxidase is the ULT drug of choice $(13,14,16,17)$. In patients that experience Allopurinol Hypersensitivity Syndrome or have a history of renal insufficiency, febuxostat, a xanthine oxidase inhibitor lacking a purine core, is metabolized in the liver $(13,14,16,17)$ and can safely be used in patients with renal impairment, is a low-cost second option for patients who are contraindicated for allopurinol. 
Table I. Long-term therapies of gout.

\begin{tabular}{|c|c|c|c|}
\hline Drug & Target & Effects & Concerns \\
\hline Allopurinol & $\begin{array}{l}\text { Purine analog xanthine } \\
\text { oxidase inhibitor }\end{array}$ & $\begin{array}{l}\text { Reduced production } \\
\text { of uric acid }\end{array}$ & $\begin{array}{l}\text { Allopurinol hypersensitivity syndrome, } \\
\text { renal impairment }\end{array}$ \\
\hline Febuxostat & Xanthine oxidase inhibitor & $\begin{array}{l}\text { Reduced production } \\
\text { of uric acid }\end{array}$ & Liver impairment, cost \\
\hline Probenecid & $\begin{array}{l}\text { Uricosuric effect via competitive } \\
\text { inhibition of URAT } 1^{\text {a }}\end{array}$ & $\begin{array}{l}\text { Decreased reabsorption } \\
\text { of urate }\end{array}$ & $\begin{array}{l}\text { Urate crystal development in urinary } \\
\text { tract, hypersensitivity }\end{array}$ \\
\hline
\end{tabular}

${ }^{a}$ URAT1 is an integral membrane protein localized to the apical (brush border) membrane of renal proximal tubular cells. URAT1 is a member of the organic anion transporter family that has been shown to mediate the transport and reabsorption of uric acid, thereby exhibiting an important role in the regulation of blood uric acid concentrations. URAT1, urate transporter 1.

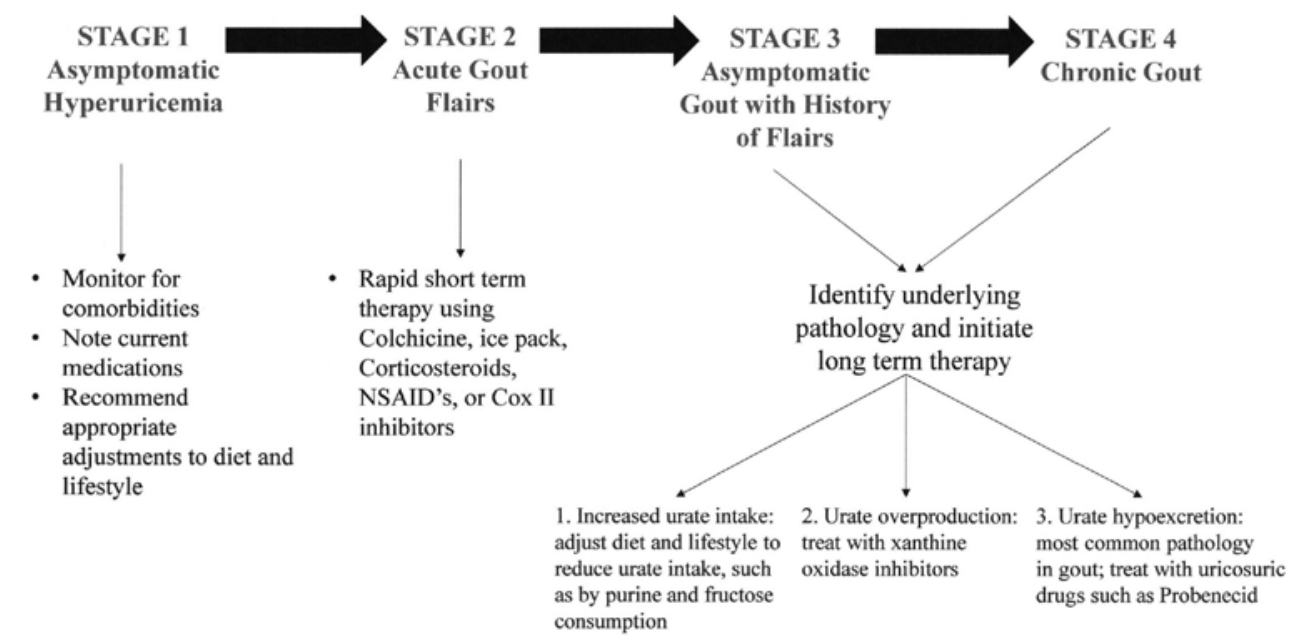

Figure 1. Stages of gout. An illustration of the progression of gout, including characteristic features and clinical treatments. As stage 1 patients are asymptomatic, few physicians select urate lowering therapy (ULT). Although there is no absolute consensus, this scheme reflects a popular criteria for ULT initiation. ULT can be subdivided into three pathological conditions, each with its own appropriate treatment.

\section{Probenecid as a uricosuric drug}

The process by which the human renal proximal tubule extensively reabsorbs urate is blocked by uricosuric drugs, including probenecid (12). It is estimated that $90 \%$ of the daily load of urate is reabsorbed, and this process is sustained by a family of organic anion transporters (OATs) including the urate transporter 1 (URAT1), which are targeted and inhibited by uricosuric drugs (18) (Fig. 2). URAT1 is a sodium-independent exchanger of chloride and organic anions including urate $(19,20)$. Therefore, probenecid can be used as a long-term therapy of gout by minimizing post-secretory reabsorption of urate in hyperuricemic patients. In addition to URAT1, probenecid inhibits other organic anion transporters, including OAT1, OAT2, OAT3 and OAT4, to varying degrees (19). While URAT1 transports urate across the luminal membrane of the renal proximal tubule, it is believed that OAT1 and OAT3 transport urate across the basolateral membrane. For probenecid to have its intended cis-inhibitory effect it must be excreted into the renal tubule. Once it is in the lumen of the renal tubule, it can interact with URAT1 (20).
According to the Four Component Hypothesis (12), transport of urate begins with glomerular filtration, reabsorption of filtered urate, secretion and post-secretory reabsorption $(12,21)$. In this mechanism, probenecid most likely reduces post-secretory reabsorption by inhibiting URAT1. Notably, urinary urate excretion was observed to increase by $\leq 84 \%$ in 47 patients with chronic renal failure, suggesting loss of function of OATs such as URAT1 that contribute to post-secretory reabsorption of urate (21). The paradoxical effect mimics that of probenecid, and therefore patients with chronic renal failure are likely contraindicated for probenecid and may have a reduced response to the drug.

Probenecid is highly lipid soluble and also strongly binds plasma proteins such as albumin (10). Humans metabolize probenecid by oxidation of alkyl side chains and to a lesser extent by glucuronide conjugation; however the oxidized metabolites have nearly equal uricosuric effects as probenecid (10). Probenecid is eliminated from the human body by the renal system, and its renal clearance is dependent on $\mathrm{pH}$ and urine flow rate (10). Maximal renal clearance of probenecid is established by alkaline urine and increasing urine flow rate. Maintaining alkaline urine also minimizes the development of 


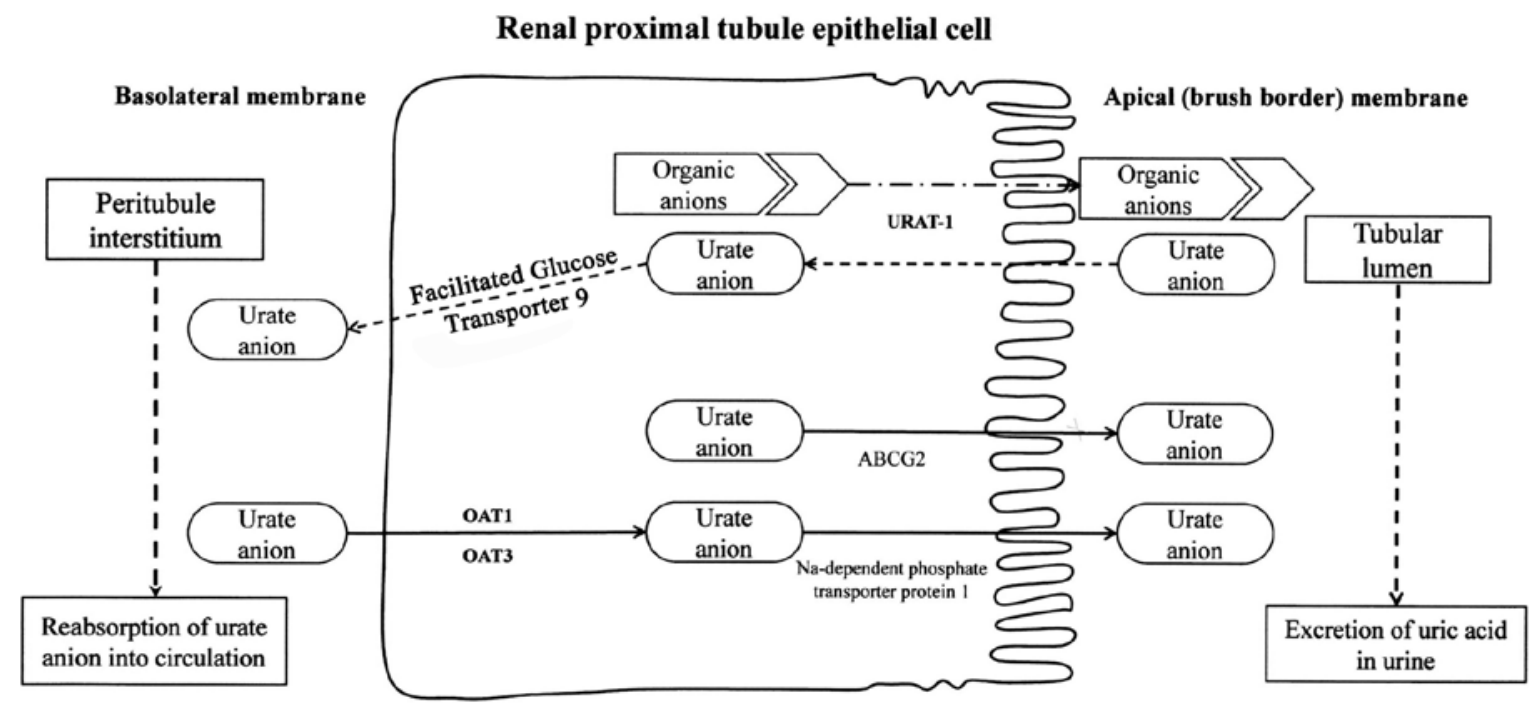

Figure 2. Renal proximal tubule epithelial cell transporters. Several transporters identified on the proximal renal tubule aid with the movement of the urate anion across epithelial cells. Urate anion crosses the basolateral membrane using the organic anion transporter 1 (OAT1) and OAT3. Thereafter, it can cross into the luminal space using the purine nucleoside transporter adenosine triphosphate-binding cassette (ABCG2) or the Na-dependent phosphate transporter protein 1. The major mechanism by which uricosuric drugs, such as probenecid, function is by competitive inhibition of urate transporter 1 (URAT1), effectively blocking reabsorption of the urate anion from the apical membrane into the basolateral membrane and circulation. Facilitated glucose transporter 9 is implicated in basolateral reabsorption of urate. This figure is a simplified illustration of the mechanisms involved in urate removal and reabsorption, and is not comprehensive. Adapted from Terkeltaub (13).

urate crystals in the urinary tract when using probenecid (22). Probenecid is preferentially administered orally, and use can result in side effects, although severe side effects are rare. The most severe side effect is probenecid hypersensitivity, which is characterized by fever, rash, nausea, vomiting and vasomotor collapse, which occurred in 8 subjects in a study observing 2,502 patients using probenecid (22). There was no observed hepatic or renal toxicity observed, and to date no fatalities have been caused by use of the compound. Gastrointestinal problems, ranging from abdominal distress and vomiting to diarrhea, appear to be the most common side effects observed (22).

When comparing their effectiveness as urate lowering drugs, there is no clear advantage of allopurinol over probenecid (23). However, allopurinol is usually preferred by physicians due to its reduced side-effect profile and higher tolerability in comparison to probenecid. In a study of 31 gout patients, it was shown that probenecid prescribed concomitantly with allopurinol increased the overall hypouricemic effect and reduced plasma urate levels by an additional $25 \%$ in comparison to allopurinol alone (24). Notably, in addition to urate, oxypurinol, the metabolite of allopurinol, is also a substrate of URAT1. Probenecid blocks post-secretory uptake of oxypurinol and reduces the efficacy of allopurinol. However, the uricosuric action of probenecid was observed to compensate for this undesired effect (24).

Based on the above evidence, probenecid is a viable option for the long-term management of gout. However, xanthine oxidase inhibitors, such as allopurinol have a smaller side-effect profile and an increased tolerability, and thus maintain their position as the primary drug of choice for gout. Thus, probenecid is generally only used in patients with refractory gout or in patients who cannot tolerate allopurinol or febuxostat. While it has been traditionally prescribed as a

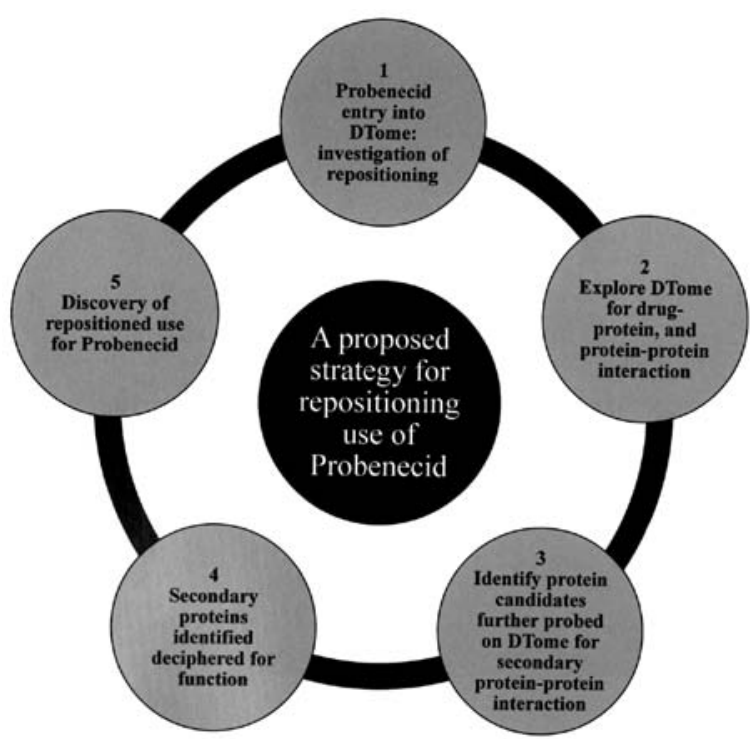

Figure 3. A proposed strategy for the repositioning use of probenecid

monotherapy, the future direction of probenecid may involve increased concomitant use with other ULT medications, such as xanthine oxidase inhibitors.

\section{Repositioning of probenecid using DTome analysis}

The observed pronounced effects probenecid exerts on OATs, coupled with the fact that OATs have a vital role in the distribution and excretion of several widely prescribed drugs prompted us to query whether probenecid may have novel targets and uses. Our approach was to use the DTome (25) database to investigate drug-drug, drug-protein and second level protein-protein interactions for probenecid (Fig. 3). These interactions were 


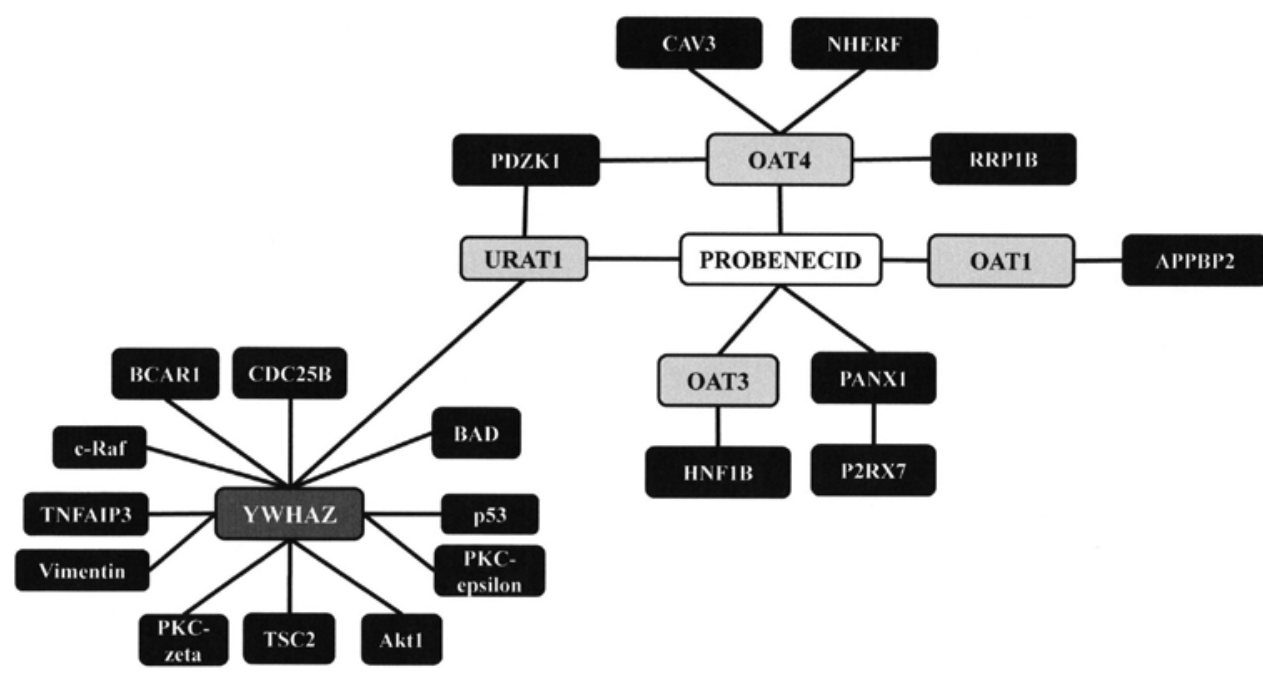

Figure 4. Drug-target interactome-based probenecid protein interactions. URAT1, urate transporter 1; OAT, organic anion transporter; PANX1, protein pannexin 1; HNF1B, hepatocyte nuclear factor 1 homeobox B; RRP1B, ribosomal RNA processing 1 homolog B; CAV3, caveolin-3; NHERF, Na $/ \mathrm{H}^{+}$exchanger regulatory factor; APPBP2, amyloid $\beta$ precursor protein binding protein 2; P2RX7, P2X purinoreceptor 7.

subsequently visualized using Cytoscape (26), a program for network analysis (Fig. 4). The network generated from the DTome results was further expanded using Cytoscape tools, which query public interaction databases. The aim was to develop a network centered on the off-target interactions of probenecid with genes involved with carcinogenesis, or altered in cancers, as well as targets that may have an anti-viral effect. Results from the DTome database showed multiple proteins for which probenecid had direct interactions. As expected, there is a direct interaction between probenecid and URAT1, a member of the SLC22 family of genes. This interaction has already been discussed as a path by which probenecid can treat gout. Additionally, there were interactions with OAT1, 3 and 4, all members of the SLC22 family of genes, as well as a fourth protein pannexin 1 (PANX1), a protein that is a structural component of gap junctions.

\section{Repositioning of probenecid as an antiviral drug}

As mentioned earlier, probenecid was used in the past to increase the plasma concentrations of medications such as penicillin when such treatments were in limited supply. There is a renewed interest in using probenecid to increase the efficacy of medications for hard to treat conditions such as human immunodeficiency virus (HIV) infection, in addition to other viral infections. While probenecid targets several different organic anion transporters, two of primary interest are SLC22A6/OAT1 and SLC22A8/OAT3 (19). In a randomized, two-way crossover study of 12 healthy subjects, probenecid was observed to competitively inhibit the reabsorption of ciprofloxacin, a quinolone antimicrobial agent. It was suggested that ciprofloxacin was competitively blocked from OAT1 by the actions of probenecid, although no conclusions could be made regarding which transporters were involved (27). While they share a degree of homology, OATs also have unexpected differences in substrate binding and transport. For example, adefovir, tenofovir, cidofovir and acyclovir all have strong affinities for OAT1 and complementary weak affinities for
OAT3 (28). Several antivirals are substrates of either OAT1 or OAT3, and thus have the potential to be manipulated by probenecid.

In addition to being in the proximal tubule of the renal system, OAT1 and OAT3 have also been localized to the modified cuboidal epithelial cells of the choroid plexus (CP) $(29,30)$. Notably, OAT3 is a sodium-dependent transporter, and has been further localized to the apical side of CP epithelial cells (31). These transporters function as an efflux mechanism to remove organic anions from the cerebral spinal fluid (CSF) into the blood. For example, the first nucleoside used in acquired immune deficiency syndrome therapy zidovudine (AZT) is rapidly removed from the CSF. This process is mediated by OATs, including OAT1 and OAT3, although the exact contributions of each OAT remain unknown (32).

Probenecid can be used to increase antiretroviral retention and alter their pharmacokinetic behavior. With the above newly discovered OATs, probenecid can achieve this by reducing loss of antivirals from the plasma into the renal system and reducing efflux of antivirals from the CSF. When combined with probenecid, blood plasma and CSF concentrations of AZT significantly increased (33). However, a previous study has suggested that severely immunodeficient patients, such as HIV patients, are more likely to experience side effects with probenecid, limiting the dose that can be safely administered. In $8 \mathrm{HIV}$ patients prescribed probenecid concomitantly with AZT, 6 experienced rashes, and 3 of those 6 had severe rashes. These patients were prescribed $500 \mathrm{mg}$ probenecid every 8 h (34).

Another study compared the efficacy and safety of a reduced dose probenecid combined with Cidofovir, an acyclic nucleotide analog of cytosine used to treat cytomegalovirus, an opportunistic infection identified in severely immunosuppressed patients, such as HIV patients. In this randomized, open-label comparison of the normal and reduced regimens of probenecid, the lower dose of probenecid combined with cidofovir delivered cidofovir without a significant change in its pharmacokinetics. The lower dose also reduced the 
likelihood of side effects (35). Based on this evidence, the pattern, timing and dose in which probenecid is administered significantly influence the tolerability and likelihood of side effects occurring. The present standard regimen of probenecid should be restructured in severely immunosuppressed patients to improve the efficacy of concomitantly prescribed drugs and minimize the occurrence of side effects. Furthermore, inhibition of an OAT, such as OAT3, by probenecid may not result in chronic symptoms, as OAT3-knockout mice did not have clear developmental defects in major organ systems (30).

Probenecid can also be used concomitantly with medications used to treat more common viral infections. Probenecid can be combined with oseltamivir, an antiviral used to treat influenza. When probenecid was prescribed four times daily, there was a significant decrease in renal excretion of oseltamivir, such that the dose of the antiviral could be decreased without compromising its efficacy (36). However, prescribing probenecid four times daily poses several challenges, including increasing the likelihood of the patient to develop side effects. Notably, in another study it was discovered that probenecid inhibits the gene expression of OAT3. The classical inhibition of OAT3 by probenecid significantly reduced the virus load of influenza A (37). It is hypothesized that by competitively inhibiting OAT3 and reducing its gene expression, probenecid attenuates the transport of certain viral factors required for infection. When probenecid was combined with oseltamivir, the virus load of influenza A was further reduced compared to probenecid alone (37).

\section{Repositioning of probenecid as an anticancer agent}

Disruptions of the PANX1 gene have been associated with melanoma tumor progression (38). The tumor progression associated with alterations in the PANX1 gene may be due to direct factors, or may be due to interaction with another protein, $\mathrm{P} 2 \mathrm{X}$ purinoceptor 7 (P2RX7). It has been shown that $\mathrm{P} 2 \mathrm{RX} 7$ is an activator of PANX1 (39); however, the two may also interact in other ways. P2RX7 has been shown to activate tumor necrosis factor- $\alpha(\mathrm{TNF}-\alpha)$, and may mediate apoptosis through that pathway (40).

Querying public databases using Cytoscape, a possible interaction was identified from a proteomics study in mice, using codetermination techniques, between URAT1 and another protein, YWHAZ. YWHAZ, also known as 14-3-3 protein $\zeta / \delta$, is a protein that mediates signal transduction by binding to phosphoserine-containing proteins (41). Modulation of signal transduction via binding to phosophoserine-containing proteins suggests that it can have a major role in modulating survival kinases. In addition to interaction with survival kinases, such as Akt (42), studies have shown that this gene interacts with the critical p53 tumor suppressor gene (43) as well as numerous other genes associated with carcinogenesis. The expression and activity of the YWHAZ protein are altered in numerous cancer cell lines. There has been little research surrounding the URAT1-YWHAZ interaction, and no research has been conducted for human cell lines. This interaction may be extremely important in identifying the off-target, chemotherapeutic properties of probenecid and could be an important area for future study.
Additional interactions that may be involved with development of cancers reported for the SLC22 family of proteins (OAT1, OAT3, OAT4 and URAT1) include CAV3, PDZK1, RRP1B, HNF1B, APPBP29 and NHERF (44). CAV3 belongs to the caveolin family of proteins serving as scaffolds for the organization of the caveolae plasma membranes, which act as reception and interface sites for signaling molecules in mammalian cells. CAV3 has also been shown to interact with EGFR to initiate events impinging on the regulation of survival kinases suggesting a significant chemotherapeutic role (45). CAV3 reportedly interacts with OAT4; however, structural domains and specific amino acid details on their interaction remain to be determined. Future studies on the molecular nature of CAV3:OAT4 binding could reveal insights on probenecid as a potential chemotherapeutic agent. Similarly, PDZK1 has been shown to bind to URAT1 and OAT4, while NHREF has been shown to bind with OAT4. Studies have shown that this association increases the activity of OAT4 and possibly URAT1 (44). Of clinical relevance is the observation that PDZK1 has a role in the regulation of breast cancer resistance proteins (46). Molecular connectivity reported to exist between RRP1B and OAT4 and other members of the SLC22 family, respectively, HNF1B with OAT3 and OAT1 with APPBP29, all point to their participatory role in carcinogenesis (25) and provide the rationale for considering the repurposing of probenecid as a drug with anticancer activities.

\section{Conclusion}

Drug repositioning is becoming an increasingly attractive choice over traditional drug development as a result of sky-rocketing cost and time of development. Three major methodologies exist for drug repositioning: Exploring drug-protein and secondary protein-protein interactions, indirect and downstream drug-ligand interactions, or drug-gene interactions using a 'Connectivity Map'. This review explored the efficacy of drug repositioning by testing drug-protein and secondary protein-protein interactions of probenecid using the DTome database. Probenecid was chosen as the focus of the present study due to decades of publicly available research and data.

The drug-protein interactions of probenecid were analyzed using DTome, and secondary protein-protein interactions were probed thereafter. The primary drug-protein interactions discovered included URAT1, OAT1, OAT3, OAT4 and PANX1. The accuracy of this methodology was supported by the presence of URAT1 in the results, which determined that a sodium-independent exchanger is involved in the post-secretory reabsorption of urate. Extensive study has proven that probenecid can block URAT1 and therefore be used as a treatment for gout.

While URAT1 is the primary target of probenecid for the treatment of gout, its other targets have been attributed with its side effects. These targets, including the OATs and PANX1, were further explored as possible avenues for drug repositioning. First, probenecid had distinct drug-drug interactions due to its interaction with OAT1 and OAT3. Specifically, it blocked reabsorption of antivirals and antimicrobials, supporting its use as an adjuvant in hard to 
Table II. Hypothesized associations between targets of probenecid and potentially-related diseases.

\begin{tabular}{ll}
\hline Primary target $^{\mathrm{a}-\mathrm{f}}$ & Associated disease process \\
\hline URAT1 $^{\mathrm{a}}$ & Gout \\
OAT1 and $3^{\mathrm{b}}$ & $\begin{array}{l}\text { General antiviral adjuvant } \\
\text { CNS infections adjuvant } \\
\text { Independent antiviral effects } \\
\text { via OAT3 }\end{array}$ \\
& Melanomas \\
PANX1 $^{\mathrm{c}}$ & Breast cancer resistance \\
URAT1-PDZK1; $^{\text {OAT4-PDZK1 }}$ & \\
URAT1-YWHAZ $^{\mathrm{e}}$ & Carcinogenesis \\
OAT4-CAV3 $^{\mathrm{f}}$ & \\
OAT4-RRP1B $^{\mathrm{f}}$ & \\
OAT3-HNF1B & \\
OAT1-APPBP2 $^{\mathrm{f}}$ & \\
\hline
\end{tabular}

${ }^{a}$ URAT 1 is as explained in Table I. ${ }^{\text {bOAT } 1}$ and 3 are OAT transmembrane proteins actively expressed in the basolateral membrane of proximal tubular cells of the kidney. They mediate the uptake of organic anions, xenobiotics and drugs from plasma into the cytoplasm of the proximal tubular cells, and their transport into the lumen of the nephrons of the kidney for excretion. 'PANX1 is a member of a protein family that comprise the structural component of the gap junctions. ${ }^{\mathrm{d} U R A T 1-P D Z K 1}$ and OAT4-PDZK1 refer to the protein-protein interactions occurring between URAT1 and its adaptor protein PDZK1 (PDZ refers to postsynaptic density 95/disc-large/zona occludens domain), and between OAT4 and PDZK1. The URAT1-PDZK1 and OAT4-PDZK1 complexes have been reported to regulate the expression of breast cancer resistance protein. ${ }^{\text {eURAT1-YWHAZ is }}$ a complex predicted by DTome. YWHAZ denotes an adaptor protein implicated in the regulation of a plethora of general and specialized signaling pathways, through the ability of YWHAZ to bind to protein partners via a phosphoserine or phosphothreonine motif. fOAT4-CAV3, OAT4-RRP1B, OAT3-HNF1B and OAT1-APPBP2 refer to protein complexes that presumably form between the respective OATs and their putative protein partners. The formed complex may have implications in carcinogenesis. CAV3 belongs to the caveolin family of proteins serving as scaffolds for the organization of the caveolae plasma membranes, which act as reception and interface sites for signaling molecules in mammalian cells. CAV3 has also been shown to interact with EGFR to initiate events impinging on the regulation of survival kinases suggesting a significant chemotherapeutic role. RRP1B was identified as a gene with profound effects on global gene transcription patterns and significant correlation with susceptibility to metastasis and prognostication for breast carcinogenesis. HNF1B is a liver-specific transcription factor harboring the homeobox-containing basic helix-turn-helic motif and having role in the transcriptional activation or suppression of genes. APPBP2 is a microtubule-interacting protein functionally linked to the transport and/or processing of the $\beta$-amyloid precursor protein and notably, also is overexpressed in breast cancer. URAT1, urate transporter 1; OAT, organic anion transporter; PANX1, protein pannexin 1; HNF1B, hepatocyte nuclear factor 1 homeobox B; DTome, drug-target interactome; RRP1B, ribosomal RNA processing 1 homolog B.

treat infections, such as with HIV. The use of probenecid can also be extended to the central nervous system as an adjuvant to maintain antimicrobial and antiviral drug CSF concentrations, which are normally rapidly depleted as a result of OAT1 and OAT3. Notably, while probenecid can be used as an adjuvant, studies have indicated that probenecid itself reduces the viral load by attenuating viral factor uptake via OAT3. Further studies are necessary to evaluate the efficacy and safety of probenecid in patients, particularly severely immunosuppressed patients.

Thus, probenecid has an significant future as a potential antiviral medication. While it can improve the efficacy and tolerability of other antivirals, probenecid itself can also contribute to antiviral treatments by blocking theorized viral factor uptake by OAT3. On a similar note, another study has discovered that multidrug-resistance protein 1 (MRP1) is localized to microglia and may contribute to the adenosine triphosphate-dependent efflux of antivirals from the CSF (47). MRP1 is another protein that can interact with probenecid, and may be another avenue for the repositioning of probenecid as an antiviral drug. However, further study is required to elucidate the contribution that channels and transporters make to the process of viral infection.

Probenecid also had several drug-protein and protein-protein interactions that prompted exploration of its use as an anticancer agent. A primary interaction with PANX1 suggested the use of probenecid in treatment of melanoma. PANX1 may contribute to cancer progression itself or by the TNF- $\alpha$ mediated pathway via its interaction with P2RX7. Notably, URAT1 also shows an interaction with YWHAZ, a signal transduction protein that interacts with survival kinases, such as Akt as well as the p53 tumor suppressor gene. While Akt and p53 are crucial to tumor progression, the URAT1-YWHAZ pathway involves several more proteins associated with carcinogenesis and collectively may be a gateway to manipulating and managing tumor growth and progression. Finally, the SLC22 family of proteins, including the OATs, have protein-protein interactions that are significant cancer pathways. The interaction of OAT4 with CAV3 is involved in downregulation of survival kinases. PDZK1 interacts with OAT4 and URAT1, and is involved with the regulation of breast cancer resistance protein. This multitude of interactions involves unique pathways among the different targets of probenecid, and in certain instances involves overlap of its targets as with the case of PDZK1. This network suggests that the diverse set of cancer-related targets of probenecid may combine synergistically in the clinical setting. However, limited clinical studies have been performed to test these pathways and more investigations are required.

The DTome analysis of the protein targets of probenecid prompted the study on the possibility of repositioning the drug as an antiviral or anticancer medication. The results were numerous and promising; not only by identifying additional targets previously considered to cause side effects such as the OATs, but also pathways already used for therapeutic use such as URAT1 showing the potential for novel clinical use through new target pathways (Table II). Repositioning is best reserved for older and perhaps outdated medicines, such as probenecid due to its ability to take maximum advantage of a wealth of readily available study and data, thus providing a good set of tools for a rapid and less expensive approach to drug development. 


\section{References}

1. Wilson JF: Alterations in processes and priorities needed for new drug development. Ann Intern Med 145: 793-796, 2006.

2. Liu PY, LeBlanc M and Desai M: False positive rates of randomized phase II designs. Control Clin Trials 20: 343-352, 1999.

3. Kaitin KI: Deconstructing the drug development process: The new face of innovation. Clin Pharmacol Ther 87: 356-361, 2010.

4. Ashburn TT and Thor KB: Drug repositioning: Identifying and developing new uses for existing drugs. Nat Rev Drug Discov 3: 673-683, 2004

5. Padhy BM and Gupta YK: Drug repositioning: Re-investigating existing drugs for new therapeutic indications. J Postgrad Med 57: 153-160, 2011

6. Campillos M, Kuhn M, Gavin AC, Jensen LJ and Bork P: Drug target identification using side-effect similarity. Science 321 : 263-266, 2008

7. Keiser MJ, Setola V, Irwin JJ, Laggner C, Abbas AI, Hufeisen SJ, Jensen NH, Kuijer MB, Matos RC, Tran TB, et al: Predicting new molecular targets for known drugs. Nature 462: 175-181, 2009.

8. Lamb J, Crawford ED, Peck D, Modell JW, Blat IC, Wrobel MJ, Lerner J, Brunet JP, Subramanian A, Ross KN, et al: The Connectivity Map: Using gene-expression signatures to connect small molecules, genes, and disease. Science 313: 1929-1935, 2006.

9. Sirota M, Dudley JT, Kim J, Chiang AP, Morgan AA, Sweet-Cordero A, Sage J and Butte AJ: Discovery and preclinical validation of drug indications using compendia of public gene expression data. Sci Transl Med 3: 96ra77, 2011.

10. Cunningham RF, Israili ZH and Dayton PG: Clinical pharmacokinetics of probenecid. Clin Pharmacokinet 6: 135-151, 1981.

11. Robbins N, Koch SE, Tranter M and Rubinstein J: The history and future of probenecid. Cardiovasc Toxicol 12: 1-9, 2012

12. Roch-Ramel F and Guisan B: Renal transport of urate in humans. News Physiol Sci 14: 80-84, 1999.

13. Terkeltaub R: Update on gout: New therapeutic strategies and options. Nat Rev Rheumatol 6: 30-38, 2010.

14. Rees F, Hui M and Doherty M: Optimizing current treatment of gout. Nat Rev Rheumatol 10: 271-283, 2014.

15. Zhu Y, Pandya BJ and Choi HK: Prevalence of gout and hyperuricemia in the US general population: The National Health and Nutrition Examination Survey 2007-2008. Arthritis Rheum 63: 3136-3141, 2011.

16. Quillen DM: Crystal arthropathies: Recognizing and treating 'the gouch'. Prim Care 37: 703-711, 2010.

17. Hamburger M, Baraf HS, Adamson TC III, Basile J, Bass L, Cole B, Doghramji PP, Guadagnoli GA, Hamburger F, Harford R, et al: 2011 recommendations for the diagnosis and management of gout and hyperuricemia. Phys Sportsmed 39: 98-123, 2011.

18. So A: Developments in the scientific and clinical understanding of gout. Arthritis Res Ther 10: 221, 2008.

19. Robertson EE and Rankin GO: Human renal organic anion transporters: Characteristics and contributions to drug and drug metabolite excretion. Pharmacol Ther 109: 399-412, 2006.

20. Enomoto A, Kimura H, Chairoungdua A, Shigeta Y, Jutabha $P$, Cha SH, Hosoyamada M, Takeda M, Sekine T, Igarashi T, et al: Molecular identification of a renal urate anion exchanger that regulates blood urate levels. Nature 417: 447-452, 2002.

21. Garyfallos A, Magoula I and Tsapas G: Evaluation of the renal mechanisms for urate homeostasis in uremic patients by probenecid and pyrazinamide test. Nephron 46: 273-280, 1987.

22. Boger WP and Strickland SC: Probenecid (benemid); its uses and side-effects in 2,502 patients. AMA Arch Intern Med 95 $83-92,1955$

23. Scott JT: Comparison of allopurinol and probenecid. Ann Rheum Dis 25 (Suppl 6): 623-626, 1966.

24. Stocker SL, Graham GG, McLachlan AJ, Williams KM and Day RO: Pharmacokinetic and pharmacodynamic interaction between allopurinol and probenecid in patients with gout. J Rheumatol 38: 904-910, 2011.

25. Sun J, Wu Y, Xu H and Zhao Z: DTome: A web-based tool for drug-target interactome construction. BMC Bioinformatics 13 (Suppl 9): S7, 2012.

26. Shannon P, Markiel A, Ozier O, Baliga NS, Wang JT, Ramage D, Amin N, Schwikowski B and Ideker T: Cytoscape: A software environment for integrated models of biomolecular interaction networks. Genome Res 13: 2498-2504, 2003.
27. Landersdorfer CB, Kirkpatrick CM, Kinzig M, Bulitta JB Holzgrabe U, Jaehde U, Reiter A, Naber KG, Rodamer M and Sörgel F: Competitive inhibition of renal tubular secretion of ciprofloxacin and metabolite by probenecid. Br J Clin Pharmacol 69: 167-178, 2010.

28. Truong DM, Kaler G, Khandelwal A, Swaan PW and Nigam SK: Multi-level analysis of organic anion transporters 1, 3, and 6 reveals major differences in structural determinants of antiviral discrimination. J Biol Chem 283: 8654-8663, 2008.

29. Nagle MA, Wu W, Eraly SA and Nigam SK: Organic anion transport pathways in antiviral handling in choroid plexus in Oat1 (Slc22a6) and Oat3 (Slc22a8) deficient tissue. Neurosci Lett 534: 133-138, 2013.

30. Sweet DH, Miller DS, Pritchard JB, Fujiwara Y, Beier DR and Nigam SK: Impaired organic anion transport in kidney and choroid plexus of organic anion transporter 3 (Oat3 (Slc22a8)) knockout mice. J Biol Chem 277: 26934-26943, 2002.

31. Sykes D, Sweet DH, Lowes S, Nigam SK, Pritchard JB and Miller DS: Organic anion transport in choroid plexus from wild-type and organic anion transporter 3 (Slc22a8)-null mice. Am J Physiol Renal Physiol 286: F972-F978, 2004.

32. Strazielle N, Belin MF and Ghersi-Egea JF: Choroid plexus controls brain availability of anti-HIV nucleoside analogs via pharmacologically inhibitable organic anion transporters. AIDS 17: 1473-1485, 2003.

33. Hedaya MA and Sawchuk RJ: Effect of probenecid on the renal and nonrenal clearances of zidovudine and its distribution into cerebrospinal fluid in the rabbit. J Pharm Sci 78: 716-722, 1989.

34. Petty BG, Kornhauser DM and Lietman PS: Zidovudine with probenecid: A warning. Lancet 335: 1044-1045, 1990.

35. Wolf DL, Rodríguez CA, Mucci M, Ingrosso A, Duncan BA and Nickens DJ: Pharmacokinetics and renal effects of cidofovir with a reduced dose of probenecid in HIV-infected patients with cytomegalovirus retinitis. J Clin Pharmacol 43: 43-51, 2003.

36. Holodniy M, Penzak SR, Straight TM, Davey RT, Lee KK, Goetz MB, Raisch DW, Cunningham F, Lin ET, Olivo N, et al: Pharmacokinetics and tolerability of oseltamivir combined with probenecid. Antimicrob Agents Chemother 52: 3013-3021, 2008.

37. Perwitasari O, Yan X, Johnson S, White C, Brooks P, Tompkins SM and Tripp RA: Targeting organic anion transporter 3 with probenecid as a novel anti-influenza a virus strategy. Antimicrob Agents Chemother 57: 475-483, 2013.

38. Penuela S, Gyenis L, Ablack A, Churko JM, Berger AC, Litchfield DW, Lewis JD and Laird DW: Loss of pannexin 1 attenuates melanoma progression by reversion to a melanocytic phenotype. J Biol Chem 287: 29184-29193, 2012.

39. Iglesias R, Locovei S, Roque A, Alberto AP, Dahl G, Spray DC and Scemes E: P2X7 receptor-Pannexin1 complex: Pharmacology and signaling. Am J Physiol Cell Physiol 295: C752-C760, 2008

40. Clark AK, Staniland AA, Marchand F, Kaan TK, McMahon SB and Malcangio M: P2X7-dependent release of interleukin-1beta and nociception in the spinal cord following lipopolysaccharide. J Neurosci 30: 573-582, 2010

41. Li Z, Peng H, Qin L, Qi J, Zuo X, Liu JY and Zhang JT: Determinants of 14-3-30 protein dimerization and function in drug and radiation resistance. J Biol Chem 288: 31447-31457, 2013.

42. Powell DW, Rane MJ, Chen Q, Singh S and McLeish KR: Identification of 14-3-3zeta as a protein kinase B/Akt substrate. J Biol Chem 277: 21639-21642, 2002.

43. Waterman MJ, Stavridi ES, Waterman JL and Halazonetis TD: ATM-dependent activation of p53 involves dephosphorylation and association with 14-3-3 proteins. Nat Genet 19: 175-178, 1998.

44. Miyazaki H, Anzai N, Ekaratanawong S, Sakata T, Shin HJ, Jutabha $\mathrm{P}$, Hirata $\mathrm{T}$, He X, Nonoguchi $\mathrm{H}$, Tomita K, et al: Modulation of renal apical organic anion transporter 4 function by two PDZ domain-containing proteins. J Am Soc Nephrol 16: 3498-3506, 2005

45. Couet J, Sargiacomo M and Lisanti MP: Interaction of a receptor tyrosine kinase, EGF-R, with caveolins. Caveolin binding negatively regulates tyrosine and serine/threonine kinase activities. J Biol Chem 272: 30429-30438, 1997.

46. Shimizu T, Sugiura T, Wakayama T, Kijima A, Nakamichi N, Iseki S, Silver DL and Kato Y: PDZK1 regulates breast cancer resistance protein in small intestine. Drug Metab Dispos 39: 2148-2154, 2011.

47. Dallas S, Zhu X, Baruchel S, Schlichter L and Bendayan R Functional expression of the multidrug resistance protein 1 in microglia. J Pharmacol Exp Ther 307: 282-290, 2003. 